\title{
Pump-probe experiments on Er coupled Si-nanocrystals rib-loaded waveguides
}

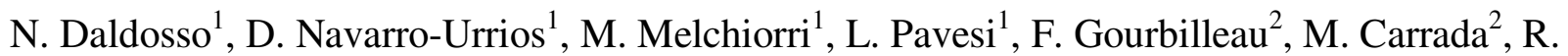
Rizk $^{2}$, C. García ${ }^{3}$, P. Pellegrino ${ }^{3}$, B. Garrido ${ }^{3}$, and L. Cognolato ${ }^{4}$

${ }^{1}$ INFM-Dipartimento di Fisica, Univ. di Trento, via Sommarive 14, 38050 Povo, Trento, Italy

${ }^{2}$ SIFCOM-ENSICAEN, 6 Boulevard Maréchal Juin, 14050 CAEN, France

${ }^{3}$ EME, Departament d'Electrònica, Universitat de Barcelona, Martí i franquès, 1, 08028

Barcelona, Spain

${ }^{4}$ Agilent Technologies, Via G. ReissRomoli 274, I-10148 Torino, Italy

\section{ABSTRACT}

Pump and probe experiments have been performed on Er-doped rib-loaded waveguides containing Si nanoclusters grown by reactive magnetron sputtering. An $\mathrm{Er}^{3+}$ absorption crosssection of about $5 \times 10^{-21} \mathrm{~cm}^{2}$ has been found at $1534 \mathrm{~nm}$ by insertion losses measurements. Transmission measurements at $1310 \mathrm{~nm}$ under optical pumping $(488 \mathrm{~nm})$ shows a decreasing of the signal because of confined carrier absorption of the Si nanoclusters. Amplification experiments at $1535 \mathrm{~nm}$ evidence two pump power regimes: losses due to confined carrier absorption in the Si nanoclusters at low pump powers and signal amplification at high pump powers. For strong optical pumping, signal enhancement of about $1.2 \mathrm{~dB} / \mathrm{cm}$ was observed.

\section{INTRODUCTION}

The convergence of photonics and microelectronics within a single chip is still suffering from the lack of a reliable on-chip optical amplifier. A possible candidate is an Er-doped planar glass optical amplifier operating at $1.5 \mu \mathrm{m}$ that can compensate for coupling and waveguide losses, and enforce split signals in optical busses. Compact optical amplifiers at $1535 \mathrm{~nm}$ with gain value of about $2-4 \mathrm{~dB} / \mathrm{cm}$ have been demonstrated by using optical pumping [1,2]. However, the gain of Er-doped planar glass optical amplifiers is limited by cooperative upconversion, which results in a non radiative de-excitation of two interacting excited $\mathrm{Er}^{3+}$ ions, and excited state absorption, in which a pump photon is absorbed by an excited $\mathrm{Er}^{3+}$ ion. Moreover, direct $\mathrm{Er}^{3+}$ excitation requires expensive $980 \mathrm{~nm}$ or $1480 \mathrm{~nm}$ lasers with quite large pump powers $(10-100 \mathrm{~mW})$. To overcome these limitations, alternative schemes have been chased. One of these is the use of suitable $\mathrm{Er}^{3+}$ sensitizers [3]. Among the various sensitizers, $\mathrm{Si}$ nanoclusters ( $\mathrm{Si}-\mathrm{nc}$ ) in $\mathrm{SiO}_{2}$ are very promising because of their broad and continuous absorption band in the visible. $\mathrm{Er}^{3+}$ ions are excited through Auger-type interaction with excitons photoexcited within the Si-nc [4]. This indirect $\mathrm{Er}^{3+}$ excitation is fast $(\sim 1 \mu \mathrm{s})$, very efficient $(>70 \%)$, with a large excitation cross section $\left(\sim 10^{-16} \mathrm{~cm}^{2}\right.$ at $\left.488 \mathrm{~nm}\right)$ [4] typical of Si-nc absorption cross section. In addition, the presence of Si-nc in the oxide increases the effective refractive index of the material and allows its use as core layer in a planar waveguide. The demonstration of efficient electroluminescent devices [5] suggests that electrical pumping of the amplifier could be feasible, which further increases the interest in the system for on-chip integration.

Encouraging results reported internal gain by LED (light emitting diode) pumping in silica waveguides doped with Si-nc and Er and also reported an unexpectedly high emission cross section for $\mathrm{Er}^{3+}$ of $\sigma_{\mathrm{em}} \sim 2 \times 10^{-19} \mathrm{~cm}^{2}$ at $1535 \mathrm{~nm}$, roughly two orders of magnitude higher than for $\mathrm{Er}^{3+}$ in pure silica [6]. Also, a report of an absorption cross section to a value of $\sigma_{\mathrm{abs}} \sim 8 \times 10^{-20} \mathrm{~cm}^{2}$ at $1535 \mathrm{~nm}$ appeared [7]. 
Here we address the problem of light amplification in Er-doped silica rib-loaded waveguides containing Si-nc grown by reactive sputtering. The effective absorption cross section of the $\mathrm{Er}^{3+}$ was measured as well as the propagation losses and signal enhancement by optical pumping in the visible.

\section{SAMPLES AND EXPERIMENTAL METHODS}

$\mathrm{Er}^{3+}$ coupled to $\mathrm{Si}-\mathrm{nc}$ rich waveguides have been prepared by reactive magnetron cosputtering of a pure silica target topped with $\mathrm{Er}_{2} \mathrm{O}_{3}$ pellets [8,9]. The incorporation of silicon excess in the film was obtained by mixing the plasma with hydrogen, owing to its ability to reduce the oxygen provided by the silica target [10]. More details on the process have been given elsewhere [8,9]. After the deposition of $0.75 \mu \mathrm{m}$ thick Er/Si-nc rich layer, a $\mathrm{SiO}_{2}$ cladding layer having a thickness of $1 \mu \mathrm{m}$ has been deposited by sputtering a $\mathrm{SiO}_{2}$ target in pure argon plasma. Then the samples have been annealed during 1 hour at $900^{\circ} \mathrm{C}$ under pure $\mathrm{N}_{2}$ flux. About 7 at. $\%$ of $\mathrm{Si}$ and an Er concentration around $N_{E r} \approx 4 \times 10^{20} \mathrm{~cm}^{-3}$ (corresponding to about 0.5 at. \%) have been determined by Secondary Ion Mass Spectroscopy (SIMS) and Rutherford Backscattering Spectroscopy (RBS) experiments. M-lines measurements allowed determining the refractive index $n$ of the film, giving a value of 1.531 at $1553 \mathrm{~nm}$. Standard processing (optical lithography and reactive ion etching) has been used to define rib-loaded waveguides with rib width ranging between 2 to $6 \mu \mathrm{m}$ and optical confinement factor $\Gamma \approx 0.55$. The wafers were cut to have $3 \mathrm{~cm}$ long waveguides that were used for all measurements.

Propagation losses in the waveguides have been obtained by measuring the insertion losses. Light from a tuneable laser $(1.5-1.6 \mu \mathrm{m})$ or a diode laser $(1.31 \mu \mathrm{m})$ was coupled into the waveguide through a single-mode polarization-preserving tapered fiber, and detected with a microscope objective (40x) matched to a zoom (2-12x) mounted on a high performance InGaAs camera and, through a prism beam splitter, was sent to a calibrated Ge detector.

Pump and probe measurements were performed in a similar setup where optical pumping was supplied by an Ar laser (488 nm, up to a power of $2.4 \mathrm{~W}$ ), focussed on the waveguide surface into a stripe $10 \mu \mathrm{m}$ wide and $0.9 \mathrm{~cm}$ long by means of a cylindrical lens. The alignment of the pump with the rib-loaded waveguide was checked by two cameras for side and top observations. Note that only the last $0.9 \mathrm{~cm}$ of the $3 \mathrm{~cm}$ long rib waveguide was pumped. The probe signal was chopped $(10 \mathrm{kHz}, 0.2 \mathrm{~mW})$ and detected through a lock-in to clean the ASE (amplified spontaneous emission) from the amplified signal.

\section{RESULTS}

Propagation losses have been determined from insertion losses measurements once the coupling losses have been estimated (about $10 \mathrm{~dB}$ ) both by calculations accounting for geometrical factors, reflection, misalignments, and by comparison with similar waveguides in which propagation losses have been measured independently [11].

Propagation loss coefficients for unpolarized light at different wavelengths are shown for $5 \mu \mathrm{m}$ wide rib-loaded in the inset of figure 1. No significant differences either in the modal profile or in the insertion losses for the TE and TM modes have been observed. The difference between losses at 1534 and $1600 \mathrm{~nm}$ is due to the $\mathrm{Er}^{3+}$ absorption and to the reduction of scattering losses with increasing wavelength. By assuming negligible $\mathrm{Er}^{3+}$ absorption at $1600 \mathrm{~nm}$, we can subtract the scattering losses as a function of the wavelength and thus estimate the $\mathrm{Er}^{3+}$ absorption contribution to the propagation losses. In figure 1 (dotted line) the result of such an estimate is reported, whereas full line is the luminescence spectrum measured in the waveguide. 
F11.3.3

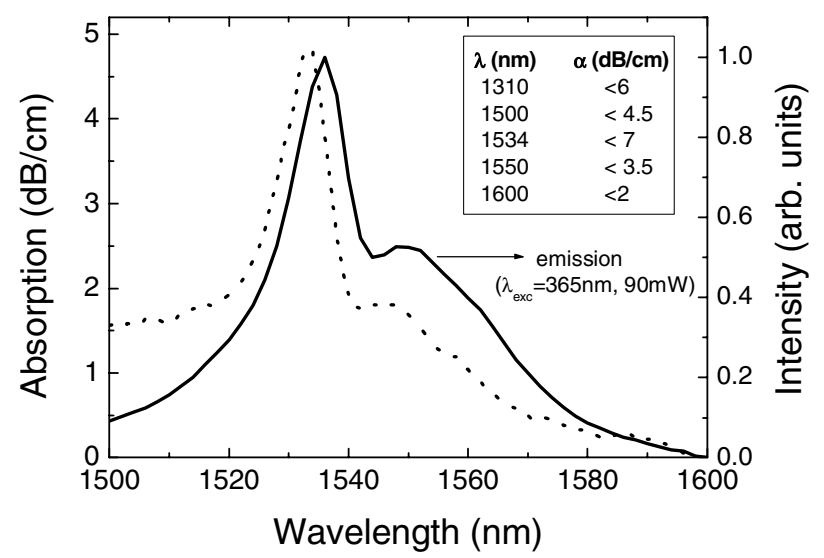

Figure 1. Emission (full line) and absorption (dotted line) spectra of Er-doped Si nanoclusters waveguide. The emission spectrum is obtained with an excitation wavelength of $365 \mathrm{~nm}$ and excitation optical power of $90 \mathrm{~mW}$. The absorption spectrum is obtained by measuring the insertion losses as a function of the wavelength. Room temperature measurements. The inset reports propagation losses coefficients for $5 \mu \mathrm{m}$ wide rib-loaded waveguide at different wavelengths.

The $\mathrm{Er}^{3+}$ absorption coefficient $\alpha_{\mathrm{abs}}=\Gamma \sigma_{\mathrm{abs}} N_{\mathrm{Er}}$ is about $4.8 \mathrm{~dB} / \mathrm{cm}$ at $1534 \mathrm{~nm}$, which corresponds to a peak $\sigma_{\mathrm{abs}} \approx 5 \pm 2 \times 10^{-21} \mathrm{~cm}^{2}$. This value coincides with that of $\mathrm{Er}^{3+}$ in pure silica $\left(4-7 \times 10^{-21} \mathrm{~cm}^{2}\right)$ [11] and contrasts to the value $\sigma_{\mathrm{abs}}=8 \times 10^{-20} \mathrm{~cm}^{2}$ measured at $1532 \mathrm{~nm}$ in Ref. [7]. Ion implantation, larger Si excess (13 at. \%), smaller $\Gamma$ factor and higher annealing temperature were used in Ref. [7]. The higher annealing temperature causes the complete crystallization of the nanoclusters in Ref. [7], whereas here the annealing temperature was chosen such that the energy transfer efficiency of the Si-nc (still amorphous) was maximized [9].

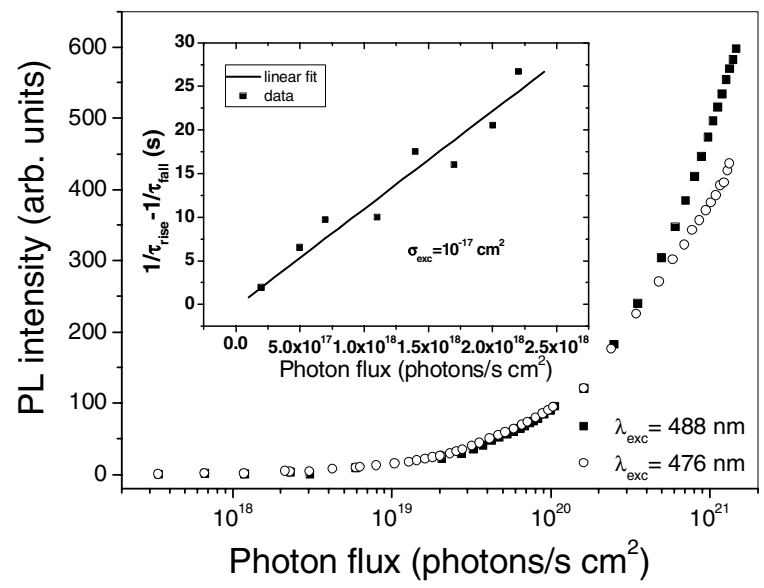

Figure 2. Luminescence intensity at $1535 \mathrm{~nm}$ as a function of the photon flux for resonant (488 $\mathrm{nm})$ and non-resonant $(476 \mathrm{~nm})$ excitations. Room temperature measurements. Inset: Er lifetimes measurements as a function of the photon flux for excitation wavelength of $476 \mathrm{~nm}$.

One may wonder whether the Si-nc actually acted as $\mathrm{Er}^{3+}$ sensitizers in our waveguides as reported in the literature. A detailed study of these samples was reported in Ref. [9] where $\mathrm{Er}^{3+}$ 
effective excitation cross sections $\sigma_{\mathrm{exc}} \sim 5 \times 10^{-16} \mathrm{~cm}^{2}$ have been found at $457 \mathrm{~nm}$. Further evidences of the coupling of the Si-nc with the $\mathrm{Er}^{3+}$ ions are reported in figure 2 . The luminescence intensity at $1535 \mathrm{~nm}$ for both resonant $(488 \mathrm{~nm})$ and non-resonant $(476 \mathrm{~nm})$ excitation as a function of excitation power has been measured. At low photon fluxes $\left(\phi<10^{19}\right.$ photon $/ \mathrm{s} \mathrm{cm}^{2}$ ) no differences are observed, which implies efficient energy transfer from Si-nc to Er ions, as shown in Ref. [9] for smaller photon flux $\left(10^{16}-10^{17}\right.$ photon/ $\left./ \mathrm{cm}^{2}\right)$. At higher fluxes $\left(\phi>10^{20}\right.$ photon $\left./ \mathrm{s} \mathrm{cm}^{2}\right)$ the emission excited by $488 \mathrm{~nm}$ pumping becomes larger than the one excited by $476 \mathrm{~nm}$ pumping due to saturation of the Si-nc excitation [13]. In this excitation regime, direct excitation of $\mathrm{Er}^{3+}$ becomes more efficient than indirect excitation through the $\mathrm{Si}$ nc.

Er lifetimes have been measured and reported in the inset of figure 2: decay times are about $3.9 \mathrm{~ms}$ and remain constant with increasing the photon flux, suggesting that both Auger and Er "concentration quenching" mechanisms (such as cooperative Er upconversion and excited state absorption) are ruled out.
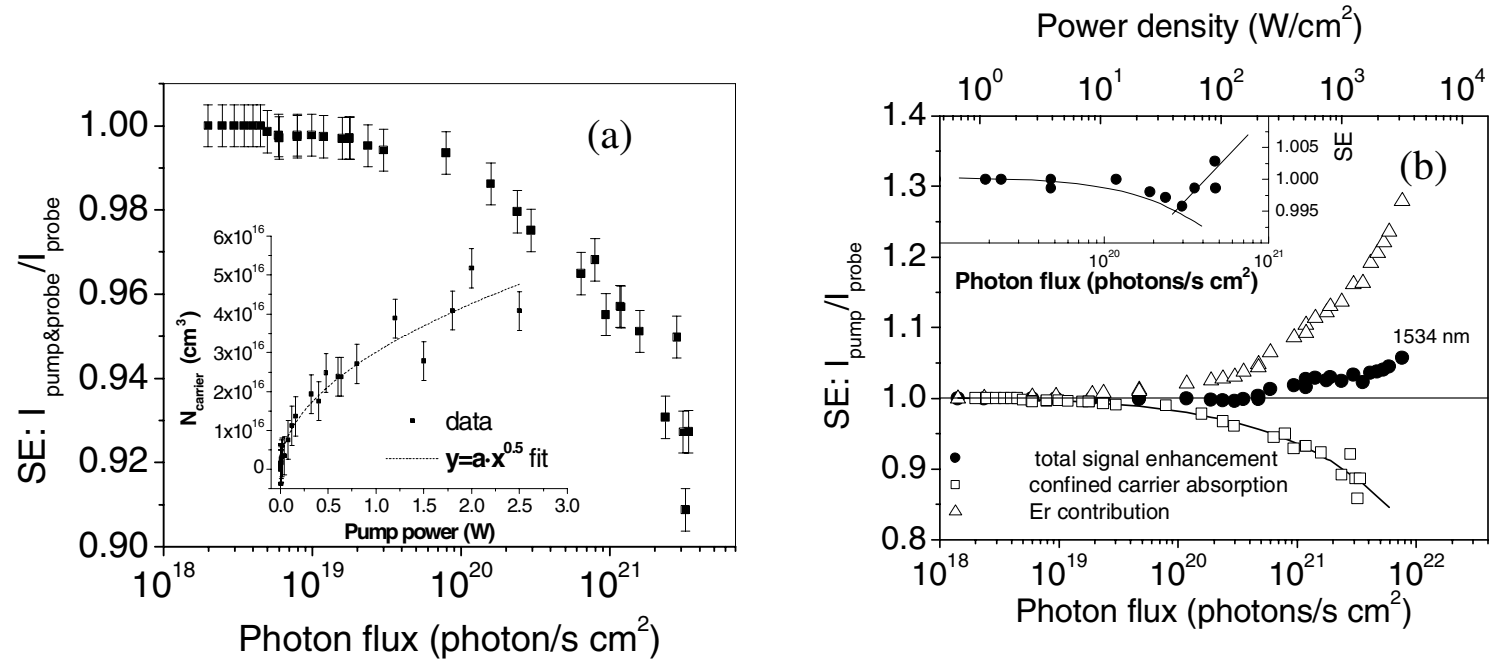

Figure 3. Signal enhancement as a function of the pump photon flux density at $488 \mathrm{~nm}$ : (a) at $1310 \mathrm{~nm}$. Inset: carrier concentration as a function of the pump power with a square root fit. (b) at $1534 \mathrm{~nm}$ (discs). Squares and triangles refer to the contribution to SE due to confined carrier absorption and $\mathrm{Er}^{3+}$ amplification, respectively. Inset: a blow-up of the SE for photon flux of $10^{19}-10^{21}$ photons $/ \mathrm{s} \mathrm{cm}^{2}$.

Pump-probe results are reported in figure 3 for signal wavelengths of $1310 \mathrm{~nm}$ (a) and 1534 $\mathrm{nm}$ (b) and pumping wavelength of $488 \mathrm{~nm}$. The transmitted signal intensity under optical pumping $I_{\mathrm{p} \& \mathrm{p}}$ can be approximated by $I_{\mathrm{p} \& \mathrm{p}} \approx I_{\text {probe }} e^{2\left(\sigma_{e m} \Gamma N^{*}{ }_{E r}\right) L},{ }^{6}$ where $I_{\text {probe }}$ is the transmitted signal intensity in absence of optical pumping, $N_{\mathrm{Er}}^{*}$ is the density of excited $\mathrm{Er}^{3+}$, and $L$ is the length of the optically pumped rib waveguide; thus the signal enhancement $S E=I_{\mathrm{p} \& \mathrm{p}} / I_{\text {probe }} . \mathrm{SE}$ measurements at $1310 \mathrm{~nm}$ allow investigating the performance of the waveguide under optical pumping in a wavelength region where $\mathrm{Er}^{3+}$ is not active. Data shown in figure 3 a reveal that $I_{\mathrm{p} \& \mathrm{p}}$ decreases with increasing the pump fluxes $\Phi$. We attribute this effect to confined carrier absorption of the signal light within the excited Si-nc [14]. Confined carrier absorption has been observed at $1534 \mathrm{~nm}$ for $\mathrm{Er}^{3+}$ in $\mathrm{Si}$-nc at high pump powers, and has two effects: it reduces the indirect excitation efficiency of the $\mathrm{Er}^{3+}$ ions via the $\mathrm{Si}-\mathrm{nc}$ and it increases the propagation losses 
at the signal wavelength [7]. If we consider, according to Ref. [7], that $\mathrm{dN}_{\text {carr }} / \mathrm{dt}=\mathrm{R}-\mathrm{CN}_{\text {carr }}{ }^{2}$ with $\mathrm{R}$ the generation rate of carriers and $\mathrm{C}\left(\mathrm{cm}^{3} \mathrm{~s}^{-1}\right)$ a proportionality constant, and assuming that the carrier generation rate is proportional to the pump power, the steady state solution gives: $\mathrm{N}_{\text {carr }}$ is proportional to power ${ }^{0.5}$. From the relation $\alpha=\sigma_{\text {carr }} \mathrm{N}_{\text {carr }} \Gamma$ (where $\sigma_{\text {carr }}$ is the free carrier absorption cross-section in bulk silicon [15]) we have determined the free carrier concentration $\left(\mathrm{N}_{\text {carr }}\right)$ within the Si nanoclusters as a function of the pump power, which shows this dependence (see the inset of figure $3 \mathrm{a}$ ).

Figure $3 \mathrm{~b}$ shows SE at $1534 \mathrm{~nm}$ as a function of $\Phi$. Two trends are clear. For low $\Phi$ (i.e. $\Phi$ $<4 \times 10^{20}$ photons $/ \mathrm{s} \mathrm{cm}^{2}$ ), a behaviour similar to the one observed at $1310 \mathrm{~nm}$ is measured (see the inset), i. e. confined carrier absorption in the Si-nc dominates. Note that the data reported in Ref. [7] refers to this low pumping regime. For higher $\Phi$, the signal is amplified and a maximum $\mathrm{SE} \approx 1.06$ for $\Phi \approx 6 \times 10^{21}$ photons $/ \mathrm{s} \mathrm{cm}^{2}$ is observed. SE has been observed only for signal wavelengths in the 1530-1540 nm range, i. e. the SE overlaps the absorption peak of $\mathrm{Er}^{3+}$ shown $^{2}$ in figure 1 .

The data reported in the inset of figure $3 b$, where a region of $S E<1$ is observed, shows that the two processes (confined carrier absorption and $\mathrm{Er}^{3+}$ related gain) can be considered additive: $\mathrm{Er}^{3+}$ are decoupled from the Si-nc at high photon flux. For this reason, we model the contribution of confined carrier absorption and subtract it from the measured SE. Considering that the free carrier absorption cross-section $\sigma_{\mathrm{fc}(1.54 \mu \mathrm{m})} \approx 1.6 \cdot \sigma_{\mathrm{fc}(1.3 \mu \mathrm{m})}$ [15], we can estimate the contribution of confined carrier absorption to SE at $1.54 \mu \mathrm{m}, \mathrm{SE}_{\mathrm{cc}(1.54 \mu \mathrm{m})} \approx \exp \left(-\alpha_{\mathrm{cc}(1.54 \mu \mathrm{m})} L\right) \approx \exp \left(-1.6 \cdot \alpha_{\mathrm{cc}(1.3 \mu \mathrm{m})}\right.$ $L) \approx\left(\mathrm{SE}_{1.3 \mu \mathrm{m}}\right)^{1.6}$. This estimate is reported in figure $3 \mathrm{~b}$ as open squares. By division of the contribution of $\mathrm{SE}_{\mathrm{cc}}$ to the measured $\mathrm{SE}$, one can isolate the $\mathrm{Er}^{3+}$ related contribution to $\mathrm{SE}$, which is plotted in figure $3 \mathrm{~b}$ as triangles. It is worth to note that confined carrier absorption is an effect dependent on the pumping and not on the probe; moreover, it introduces a loss mechanism but also a turning off of the indirect channel of $\mathrm{Er}^{3+}$ excitation so that $\mathrm{Er}^{3+}$ is mostly excited through direct absorption. Indeed experiments performed by optically pumping the system at 457 $\mathrm{nm}$ (non-resonant wavelength for $\left.\mathrm{Er}^{3+}\right)$ do not show any SE up to $250 \mathrm{~mW}\left(\boldsymbol{\Phi}=1.4 \times 10^{20}\right.$ photons $/ \mathrm{s} \mathrm{cm}^{2}$ ). No SE $>1$ has been measured by optically pumping at $532 \mathrm{~nm}$ up to $\Phi=2 \times 10^{21}$ photons $/ \mathrm{s} \mathrm{cm}^{2}$. It is worth to be noted that $532 \mathrm{~nm}$ excitation is not resonant with Er levels, thus confirming that Si-nc are not acting as sensitizers because of the presence of confined carrier absorption, which becomes significant for $\Phi>10^{20}$ photons $/ \mathrm{s} \mathrm{cm}^{2}$. In addition, an evaluation of $\sigma_{\text {exc }}$ from the SE data following the effective 2 level model of Ref. [6] yields $\sigma_{\mathrm{exc}} \sim 6 \times 10^{-21} \mathrm{~cm}^{2}$ at $488 \mathrm{~nm}$ for high photon flux. This value is rather far from the value obtained in Ref. [9] and the commonly accepted one for the $\mathrm{Er}^{3+} / \mathrm{Si}$-nc coupled system [4]. From the maximum effective SE of 1.28 due to $\mathrm{Er}^{3+}$ it is possible to estimate the number of Er ions in the excited state: as $\ln (S E)=2 \Gamma N_{E r}^{*} \sigma_{e m} L$ and $\sigma_{\mathrm{em}} \approx \sigma_{\mathrm{abs}}$, we obtain $N_{\mathrm{Er}}^{*}=5 \times 10^{19} \mathrm{~cm}^{-3}$, i. e. about $12.5 \%$ of $\mathrm{Er}^{3+}$ is in the excited state. In Ref. [6], a larger $g \sim 7 \mathrm{~dB} / \mathrm{cm}$ has been reported for a $2.5 \mu \mathrm{m}$ thick waveguide with only 1 at. \% Si excess and 0.03-0.05 at. \% of $\operatorname{Er}\left(N_{\mathrm{Er}} \approx 1-\right.$ $\left.2 \times 10^{19} \mathrm{~cm}^{3}\right)$. The use of a very low Si excess and of a short annealing time $\left(5^{\prime}\right.$ at $\left.1000{ }^{\circ} \mathrm{C}\right)$ reduced the strength of confined carrier absorption, kept effective the indirect excitation of $\mathrm{Er}^{3+}$, and could explain the high $g$. From the data, an enhanced $\sigma_{\mathrm{em}} \sim 2 \times 10^{-19} \mathrm{~cm}^{2}$ at $1535 \mathrm{~nm}$ was deduced, which is surprising [6]. In fact, the Einstein relation relates $\sigma_{\mathrm{em}}$ to the inverse of the radiative lifetime $\tau_{\text {rad }}$ of $\mathrm{Er}^{3+}$ : an increase of $\sigma_{\mathrm{em}}$ should correspond to a decrease of $\tau_{\text {rad. This was }}$ not observed and a luminescence decay time of about $8 \mathrm{~ms}$ were reported in Ref. [6]. 


\section{CONCLUSIONS}

Insertion losses and pump and probe measurements on Er dope Si nanoclusters rib-loaded waveguides have shown that at high photon fluxes a sizeable signal enhancement was present, together with a simultaneous lack of enhancement in the absorption cross section of $\mathrm{Er}^{3+}$ coupled to Si-nc, and a strong negative role of confined carrier absorption in the Si-nc, which turn off their sensitizer action. In order to obtain efficient all-optical amplifiers with the $\mathrm{Er}^{3+} \mathrm{Si}$-nc system the right route could be to decrease significantly the Si excess so to exploit the sensitizer effect of Si-nc and avoid confined carrier losses. Unfortunately we were not able to confirm the order of magnitude increase in the $\mathrm{Er}^{3+}$ emission cross section when Si-nc are present.

\section{ACKNOLEDGEMENTS}

We thank E. Chierici and E. Emelli for waveguide processing and characterization, and C. Sada for the RBS and SIMS measurements. This work has been supported by EC through the SINERGIA program. One of the authors (M. Carrada) would like to thank the Region Basse Normandie for its financial support. The Trento work was also supported by PAT through the PROFILL project and by FIRB project.

\section{REFERENCES}

1. G. N. van den Hoven, A. Polman, C. van Dam, J. W. M. van Uffelen, and M. K. Smit, Appl. Phys. Lett. 68, 1886 (1996).

2. Y. C. Yan, A. J. Faber, H. de Waal, P. G. Kik, and A. Polman, Appl. Phys. Lett. 71, 1818 (1997).

3. A. Polman and F.C.J. M. van Veggel, J. Opt. Soc. Am. B 21, 871 (2004).

4. F. Priolo, G. Franzò, D. Pacifici, V. Vinciguerra, F. Iacona, and A. Irrera, J. Appl. Phys. 89, 264 (2001).

5. F. Iacona, D. Pacifici, A. Irrera, M. Miritello, G. Franzò, F. Priolo, D. Sanfilippo, G. Di Stefano, and P.G. Fallica, Appl. Phys. Lett. 81, 3242 (2002).

6. H. S. Han, S. Y. Seo, and J. H. Shin, Appl. Phys. Lett. 79, 4568 (2001); H. S. Han, S.

Y. Seo, J. H. Shin, and N. Park, Appl. Phys. Lett. 81, 3720 (2002).

7. P. G. Kik and A. Polman, J. Appl. Phys. 91, 534 (2002).

8. F. Gourbilleau, C. Dufour, M. Levalois, J. Vicens, R. Rizk, C. Sada, F. Enrichi, and G. Battaglin, J. Appl. Phys 94, 3869 (2003).

9. F. Gourbilleau, M. Levalois, C. Dufour, J. Vicens, and A. Rizk, J. Appl. Phys. 95, 3717 (2004).

10. C. Ternon, F. Gourbilleau, X. Portier, P. Voivenel, and C. Dufour, Thin Solid Films 419, 5 (2002).

11. N. Daldosso, M. Melchiorri, F. Riboli, M. Girardini, G. Pucker, M. Crivellari, P. Bellutti, A. Lui, and L. Pavesi, IEEE Journal of Lightwave Technology 22, 1734 (2004).

12. W. J. Miniscalco, J. Lightwave Technol. 9, 234 (1991).

13. M. Wojdak, M. Klik, M. Forcales, O.B. Gusev, T. Gregorkiewicz, D. Pacifici, G.

Franzò, F. Priolo, and F. Iacona, Phys. Rev B 69, 233315 (2004).

14. D. Pacifici, G. Franzò, F. Priolo, F. Iacona, and L. Dal Negro, Phys. Rev. B 67, 245301 (2003).

15. W. Spitzer and H.Y. Fan, Phys. Rev. 108, 268 (1957). 